Neurosurg Focus 4 (6):Article 8, 1998

\title{
The Glioma Outcomes Project: a resource for measuring and improving glioma outcomes
}

\section{Frederick A. Anderson, Jr., Ph.D., and The Glioma Outcomes Project Advisory Board Center for Outcomes Research, University of Massachusetts Medical Center, Worcester, Massachusetts}

The author describes the Glioma Outcomes (GO) Project which conducts outcomes research and develops educational programs to benefit patients who undergo surgery for glioma. In January 1997 an advisory board of neurosurgeons, neurooncologists, and clinical research scientists was formed to establish the policies governing this project and to control the dissemination of aggregate data on clinical practices and outcomes. This voluntary database is designed to 1) guide the development of educational programs to improve the care of patients and 2) provide a mechanism by which physicians can evaluate the impact of their diagnostic and therapeutic decisions in a manner that is timely, confidential, and objective.

\section{Key Words * glioma * health outcomes assessment * database * registry}

Glioma is a rare disease. Existing institutional databases represent the experience of individual surgeons or clinical centers. Local and national tumor registries collect clinical data from retrospective chart reviews. Tumor registries do not follow patients over the course of their disease or provide information from their perspective such as self-reported quality of life and satisfaction with care. A 1989 survey of practices in the care of patients with brain tumor provided valuable insight,[3] but there are no recent or ongoing databases to provide data regarding changes in clinical practice and patient outcomes.

\section{DESCRIPTION OF GO PROJECT}

The Glioma Outcomes (GO) Project was created as a pilot study to test the feasibility of addressing these issues through a North American, physician-directed, cooperative registry of patients who undergo surgery for glioma. The GO Project Advisory Board (Appendix 1) had its first meeting in January 1997, and the first patient was enrolled in October 1997. The Advisory Board will evaluate the feasibility and value of this project based on data from 400 patients; this enrollment is expected by September 1998. A 2-year follow up will be completed in September 2000. It is anticipated that this North American database of glioma patient outcomes will provide valuable data for the evaluation and improvement of care in patients with glioma. In addition, this database will provide an opportunity to track trends in glioma care, including natural history, risk factors, diagnostic approaches, resource utilization, and treatment methods. Key objectives are to: 1) identify opportunities to improve the quality of care for 
glioma patients; 2) describe diagnostic and treatment strategies and patient outcomes; 3) provide physicians with data to evaluate and improve their practices; and 4) develop hypotheses for future clinical trials. Improved outcomes for glioma patients will be promoted by publishing key findings derived from the database and by developing and disseminating educational materials for both physicians and patients.

Background. Most physicians have little or no opportunity for ongoing, objective feedback about the relationship between their patterns of clinical practice and patient outcomes. Moreover, physicians may change treatment protocol in response to anecdotal information, such as a particularly good, or bad, outcome in an individual patient, which may not be representative of their overall clinical experience. Clearly, physicians need, but seldom have access to, benchmark data that allow comparison of individual practice patterns and outcomes with the experiences of their peers in caring for comparable patients.

The Outcomes Movement. The outcomes movement was founded on the principle that patient care will improve if physicians are provided with timely and credible information linking local clinical practices and patient outcomes. Health outcomes research combines features of clinical research, continuing medical education, and quality assurance. Patient outcomes can be characterized in terms of a number of dimensions, including clinical factors, death, disease, functional status, well being, satisfaction, and cost.

It is increasingly important for neurosurgeons to demonstrate that the care they provide leads to superior outcomes, particularly in comparison with care rendered by other practitioners. Payers are shifting the burden of healthcare costs to hospitals and providers, and hospital administrators are under intense pressure to reduce costs. Increasingly, this is being accomplished by limiting access to specialists. Without objective data to substantiate superior clinical outcomes, payers and hospital administrators may insist that glioma care be provided by lower-cost providers and may limit patient access to specialists.

The GO Project provides a unique opportunity to improve the care of patients with glioma through routine monitoring of health-related outcomes combined with regular feedback to physicians. In rare diseases, adequate sample size is more easily achieved through multicenter collaborative research. Individual neurosurgeons or clinical sites seldom see a sufficient number of patients to develop the cohort of glioma patients necessary to adequately define the relationship between clinical practices and patient outcomes. Additionally, local databases cannot provide comparative benchmarks to regional and national practices and outcomes. Individual hospitals have been reluctant to devote significant resources to analysis of health outcomes, particularly for rare conditions. This provides a strong rationale for providing neurosurgeons with a voluntary, North American database to assess glioma outcomes.

Education. The GO Project Advisory Board will use the findings from the database to monitor practices in the care of glioma patients. The Advisory Board will regularly compare current clinical practices with the latest findings from controlled clinical trials and consensus recommendations.[2] When clinical practices are found to be less than optimum, feedback will be provided to neurosurgeons through newsletters, journal articles, and presentations at regional and national meetings. When large variations in practices are observed without a scientific basis to advise physicians concerning the optimum practice, the GO Project Advisory Board will apprise the research community of the need for more controlled clinical trials in these areas.

Study Protocol. Patients who undergo glioma surgery are asked to give written informed consent to participate in the GO Project in the early postsurgical period (after tumor pathology has been confirmed). Neurosurgeons complete standard data forms for their patients within 3 weeks postcraniotomy. It is often 
possible for a nurse or physician's assistant to collect these data, which usually requires 10 to 15 minutes. Additional self-reported outcomes are obtained using patient surveys. Patient self-reported data are collected during the perioperative period (usually at the first postoperative clinic visit) and at 3-month intervals thereafter. Follow up is completed at either 24 months postsurgery or death. To be enrolled in the GO Project patients must have recently undergone either a biopsy alone or a first or second craniotomy for one of the following types of brain tumor: glioblastoma multiforme, anaplastic astrocytoma, anaplastic oligodendroglioma, mixed anaplastic oligo/astrocytoma, or other anaplastic glioma.

Patient self-reported data allow assessment of important outcomes, including changes in functional capacity, general health status (the Short-Form-36 Health Survey),[4] and rates of complications. The Glioma Outcomes Questionnaire (Appendix 2), a validated set of 19 glioma-specific questions based on the Functional Assessment of Cancer Therapy-Brain instrument,[5] is included as part of the patient questionnaire. Sociodemographic and related patient characteristics are also collected to allow stratification of cases into important subgroups such as age, race, and marital status.

Approximately 10 to 12 weeks after surgery, the patient receives a Patient Follow-Up Form by mail from the Data Coordinating Center, as well as a blank Physician Follow-Up Form with instructions to bring this to their next doctor visit, a stamped preaddressed envelope, and an introductory letter to give to the physician who has assumed responsibility for their care. This letter requests the physician's assistance in gathering interval clinical data for the study. Patients also receive a plastic wallet card, identifying their enrollment in the GO Project, that provides the name and telephone number of the neurosurgeon who performed their craniotomy.

If the patient does not return the Patient Follow-Up Form within two weeks, someone from the Data Coordinating Center will call to inquire about their willingness to continue in the study. This procedure is repeated every 3 months for 2 years unless the patient dies, withdraws from the study, or is lost to follow up.

Design Limitations. A number of limitations are evident in the design of this project, some of which are inherent in the study design whereas others are uncertainties that will be clarified based on our experience in this pilot project. If and when this pilot project is expanded, it is anticipated that some of these limitations may be at least partially resolved by additional quality control measures. From the beginning, concerns about the feasibility and value of these data were raised by Advisory Board members and by a number of neurosurgeons who expressed interest in participating. Although some concerns are inherent in an observational research study design (for example, no control groups), other concerns, such as compliance of physicians and patients, can only be resolved by conducting a pilot study. The following three areas are important concerns in a voluntary outcomes registry.

1) In using an observational study design researchers cannot compare the efficacy of glioma treatments. There are no formal control groups in the GO Project. Patients are not randomly assigned to treatments. Thus, testing hypotheses regarding the comparative efficacy of treatments for glioma is not feasible. On the other hand, randomized controlled trials (RCTs) also have important limitations that may be avoided in an observational database. For example, in an attempt to provide homogeneous comparison groups, RCTs mandate a strict treatment protocol for all patients. Additionally, RCTs often use restrictive inclusion/exclusion criteria, which may lead to concerns about the generalizability of RCT findings to the often large proportion of patients in routine clinical practice who do not meet these criteria. On the other 
hand, in an observational registry it may be feasible to enroll consecutive patients with comparatively few exclusions (for example, diminished mental capacity to give consent or answer questions). Observational study designs also allow researchers to observe patient care in a natural environment (for example, no care protocols are proscribed and routine unconstrained clinical practices can be documented). Thus, compared with RCT data, GO Project data may be more representative of the full range of patients who undergo craniotomy for glioma, as well as of routine clinical practices in glioma care.

2) Compliance of a voluntary study group, including both physicians and patients, is uncertain and data may be missing, erroneous, or incomplete. A key objective of the GO Project is to test the feasibility of creating a low-cost, voluntary outcomes registry. No monetary support is given to study sites. Initially, it was not known whether patients and physicians would complete interval data forms. However, our early experience has been encouraging, particularly with respect to patient compliance.

3) Will study findings be generalizable? The 47 participating hospitals and centers and 99 neurosurgeons may not be representative of North American practice. It is not known whether the participating academic and community sites are representative of North American hospitals, surgeons or of patients who undergo surgery for glioma. It is also unknown whether the geographic distribution of surgeons and patients is representative. Although we have requested that consecutive patients be enrolled, no funds are budgeted to audit hospital discharge lists or patient charts. Thus, at least in this pilot study of 400 patients, it will not be feasible to answer these questions. However, comparable data for a number of key variables are available in tumor registries such as the Central Brain Tumor Registry of the United States,[1] and these data will be compared with findings from the GO Project database. This will allow indirect assessment of the similarity of GO Project data against a broader population that is reasonably representative of North American patients.

Maintenance of the Database. A Data Coordinating Center has been established in the Center for Outcomes Research at the University of Massachusetts Medical Center. The Data Coordinating Center prepares monthly reports for participating clinics, provides scientific support for data analysis, and promotes the publication of aggregate North American glioma outcomes.

Confidentiality of Participants. The GO Project Data Analysis Center at the University of Massachusetts Medical Center has been entrusted with the responsibility to protect the rights and confidentiality of patients, physicians, and hospitals participating in the project. Provisions for the protection of patients have been reviewed and approved by the Institutional Review Board at the University of Massachusetts Medical Center. Participating neurosurgeons must obtain approval from their local hospital Institutional Review Board. Patients must sign an approved informed consent to participate in this project.

Authority and Governance. Acting as trustee for participating physicians and their patients, the GO Project Advisory Board retains all authority for the rules governing the operation of this project, including the dissemination of aggregate findings and development of educational materials. The Advisory Board is comprised of clinical scientists, neurosurgeons, and neurologists with a special interest in glioma treatment (Appendix 1). The Board is independent and hopes to work cooperatively with all parties interested in advancing the care of glioma patients, including, but not limited to, patient advocacy groups and professional societies. The GO Project has been endorsed by the Joint Section on Tumors of the American Association of Neurological Surgeons/Congress of Neurological Surgeons. 
Data Collection Instruments. Samples of data collection instruments are available on the GO Project Web site (www.glioma.org). All data forms are color coded and customized as an aid to administration. The Data Coordinating Center prints a supply of the following data collection forms that are customized for each clinical site: 1) Enrollment Form (white), 2) Perioperative Form (blue), 3) Physician Follow-Up Form (yellow), 4) Retrospective Form (brown), 5) Initial Patient Form (ivory), 6) Patient Follow-Up Form (green), and 7) Change in Status Form (grey).

The first data collection form used is the Enrollment Form, which is filled out by the physician or the designated data-collection coordinator, and provides the Data Coordinating Center with early notification of the enrollment of a new patient. This data form is used to prepare the first mailing to the patient, containing the Physician and Patient Follow-Up Forms and the patient's GO Project identification card.

There are three types of data forms for physicians. The Perioperative Form asks physicians to detail their management of the patient at the time of surgery, including medications, type of surgery, preoperative symptoms, and early postoperative clinical findings. The Retrospective Form is completed for patients who underwent previous surgical treatment of their tumors. This provides an opportunity to gather data about clinical factors from the earlier operation so that patients enrolled at second surgery can be compared with patients enrolled at first surgery (or patients enrolled at biopsy). The Physician Follow-Up Form is completed by the physician providing glioma care at 3-month intervals following the enrollment surgery.

There are two types of data forms for patients. The Initial Patient Form is completed by the patient or, if the patient is not capable of completing the form, by a caregiver or the data-collection coordinator, who asks the patient the questions and records the answers. The Initial Patient Form is a self-administered questionnaire that solicits responses about health status, expectations, satisfaction with care, and quality of life. The Patient Follow-Up Form is used to assess changes in patient self-reported health status at 3-month intervals.

The Change in Status Form is used by the enrolling physician to report changes in the status of enrolled patients to the Data Coordinating Center, including death, change of address, or withdrawal from the GO Project.

Current Status. As of June 1, 1998, there were 47 clinic sites and 150 patients enrolled in the GO Project (Appendix 3).

Future Plans. Provided that this pilot database in 400 patients is judged both feasible and useful by neurosurgeons and patients, the GO Project will be expanded with the objective of establishing a North America-wide, multicenter, cooperative registry of glioma outcomes. Broader participation by neurosurgeons will be needed to provide representative data to assess regional differences in practices and patient outcomes.

\section{CONCLUSIONS}

The GO Project is an innovative program that appears destined to improve the care of patients. The long-term goal is to ensure a uniformly high standard of care for all patients. By helping neurosurgeons to obtain data on their practice patterns and patient outcomes, we hope to help them to monitor their patterns of care, compare recent practices with those of other neurosurgeons from across North America, and improve patient care. 


\section{References}

1. CBTRUS: 1996 Annual Report. Chicago: Central Brain Tumor Registry of the United States, 1997

2. Davies E, Hopkins A: Good practice in the management of adults with malignant cerebral glioma: clinical guidelines. Br J Neurosurg 11:318-330, 1997

3. Mahaley MS Jr, Mettlin C, Natarajan N, et al: Analysis of patterns of care of brain tumor patients in the United States: a study of the Brain Tumor Section of the AANS and the CNS and the Commission on Cancer of the ACS. Clin Neurosurg 36:347-352, 1990

4. Ware JE Jr, Sherbourne CD: The MOS 36-item short-form health survey (SF-36). I. Conceptual framework and item selection. Med Care 30:473-483, 1992

5. Weitzner MA, Meyers CA, Gelke CK, et al: The functional assessment of cancer therapy (FACT) scale. Development of a brain tumor subscale and revalidation of the general version (FACT-G) in patients with primary brain tumors. Cancer 75:1151-1161, 1995

Manuscript received April 15, 1998.

Accepted in final form June 3, 1998.

Address reprint requests to: Frederick A. Anderson, Jr., Ph.D., Center for Outcomes Research, S3-716, University of Massachusetts Medical Center, Worcester, Massachusetts 01655-0336. email: fred.anderson@ummed.edu.

This work was supported by a grant from Rhône-Poulenc Rorer, Inc.

Note: Physicians interested in obtaining additional information may call the GO Project Data Coordinating Center at (888) 820-7171.

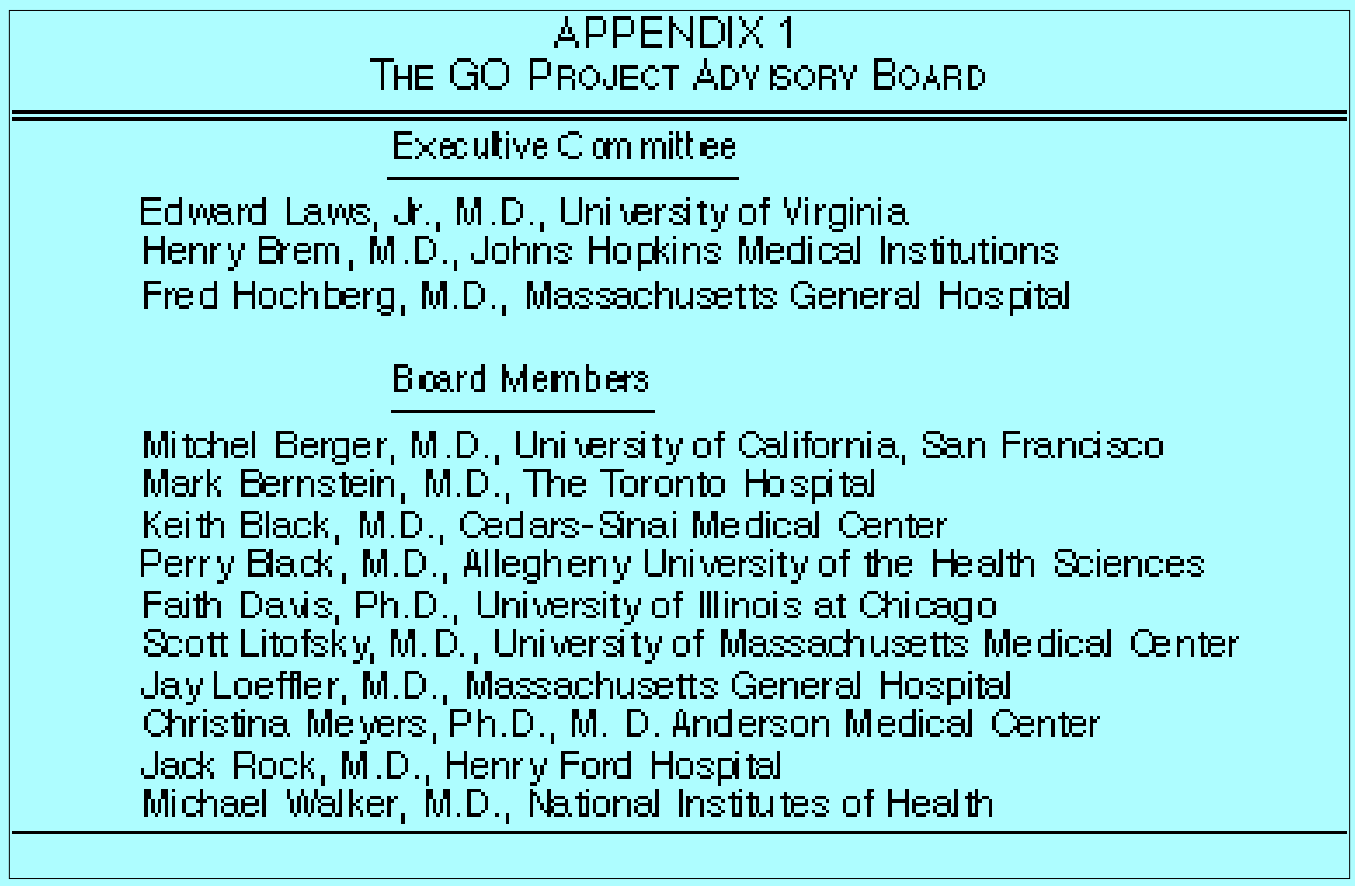




\section{APFENDIX 2}

\section{GLIOML OUTCOMES OUIESTIONNAIRE}

1. Fl ease indicate how you felt during the past wed (chocse one answer on each line)

Not Some oute very at all what a tit

much

a. I can rememter new things

b. I have troutle with my dision

c. I am atle to find the right word(s) to say what I mean

d. I have troutle expressing my thoughts

e. I am able to put my thoughts in to action

f. My personadity has ofanged

g. I haie meaknes in some parts of m y body

h. I have troutle with my coordination

i. I have had seizures

i. I have had headacties

k. I get tred easily

I. I am slomer to do things

m. I feel sick

n. I spend time in ted

o. I need hel p caring for m yself (tathing, dressing, eating, etc)

p. I get support from m y fanil y

q. I feel sad

r. I am atie to work (indude work in home)

s. I am atle to drive

t. I am atle to enjoym yusual leisure pursuits

u. I am content wij the quality of my'life

$\begin{array}{llll}\square & \square & \square & \square \\ \square & \square & \square & \square \\ \square & \square & \square & \square \\ \square & \square & \square & \square \\ \square & \square & \square & \square \\ \square & \square & \square & \square \\ \square & \square & \square & \square \\ \square & \square & \square & \square \\ \square & \square & \square & \square \\ \square & \square & \square & \square \\ \square & \square & \square & \square \\ \square & \square & \square & \square \\ \square & \square & \square & \square \\ \square & \square & \square & \square \\ \square & \square & \square & \square \\ \square & \square & \square & \square \\ \square & \square & \square & \square \\ \square & \square & \square & \square \\ \square & \square & \square & \square \\ \square & \square & \square & \square \\ \square & \square & \square & \square\end{array}$


APFENDIX 3

PARTKIFATING CLINICAL SITES

\begin{tabular}{|c|c|c|}
\hline Sit e Name & City & State'Provine \\
\hline Al tany-Troy Neurosurgical Associates, F.C. & Äl tarny & W \\
\hline Euffalo General Hospital & Euffialo & WY \\
\hline California Facific Medical Center & San Francison & Сम \\
\hline Carolina Neurosurgery \& Spine Associates & Charlotte & NO \\
\hline Cedars-Snaj Neurossingical Institute & Los unngeles & Оमे \\
\hline Decatur Muemorial Hospital & Decatur & IL \\
\hline Florida Neurosurgery' F.A. & 'ilúnter Fark & $\mathrm{FL}$ \\
\hline Geness Healthcare System & Zanesulle & $\mathrm{OH}$ \\
\hline Genesys Weurosurgical मेsouciates & Grand Earo: & |ill \\
\hline George 'ibsshington Lni iersty Medical Center & injashington & DO \\
\hline Guthrie Oinic, Ltt. & Sayte & Рவ் \\
\hline Henry' Ford Hospital & Detroit & $|\omega i|$ \\
\hline Indiana Unitersity luledical Center & Indianapolis & IN \\
\hline Inowa Institute of Fesearch and Education & Falls Chumch & 'Ud \\
\hline lo whe Health System & Des loines & Iम́ \\
\hline Johns Hopkins Hospital & Eal tim ore & MD \\
\hline Kajoer Permanente Medical Center & Sacramento & कम \\
\hline Louisana State Lniversity, School of Medicine & Ghe we port & டவ் \\
\hline lul. D. Mnderson Cancer Center & Houston & $\mathrm{TX}$ \\
\hline Medical Ini uersity of South Carolina. & Charleston & 80 \\
\hline mississppi Eaptist Health System & Jack son & MOS \\
\hline Mount Snaj Medical Center & Müiani Eeach & $\mathrm{FL}$ \\
\hline Weurossienoe Goup of Wortheast 'obsonsin & д̈ppleton & 'bì' \\
\hline Wew Jersey Neuroscience Institute & Edison & W.J \\
\hline Worth westem Iniversity medical School & Evariston & IL \\
\hline Our Lady of Loundes Muledical Center & Fhiladelphia & Fम̈ \\
\hline Fosmell Park Cancer Institute & Euffialo & W' \\
\hline FushFrestyterian-St.Luke's medical Center & Chicago & $\mathrm{IL}$ \\
\hline Salem Hospital & Salem & OFi \\
\hline Southeastem Weurosurgical \& Spine Institu te, F. H. & Green wille & $\mathrm{SO}$ \\
\hline 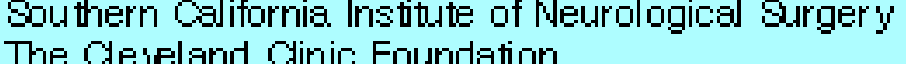 & Escondido & कम \\
\hline 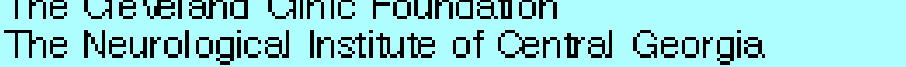 & $\begin{array}{l}\text { Ue velang } \\
\text { Macon }\end{array}$ & Gin \\
\hline The Neurosurgical Goup of Chattanooga, F.C. & Chattanooga. & TW \\
\hline The Toronto Hospital - inie stern Di usion & Toronto & ON, Carada. \\
\hline Tri-State Neuroscience Center & Huntington & 'bin' \\
\hline Uni uersity of California-San Francison & San Francison & कम \\
\hline Uni tersty of Chicapo & Chicapo & IL \\
\hline Uni uersty of Colorado Heal th Scienoes Center & Denver & 0 \\
\hline Uni uersty of llinois-P'eoria. & Péria & IL \\
\hline Uni uersity of Massactusetts luledical Center & intorcester & (冈ㅣㅁㅕ \\
\hline Uni ersity of Minnesota & Minneapolis & MN \\
\hline Uni uersity of Misouri-Colum tia. & Colum tia. & MO \\
\hline Uni 'ersity of New Mesoo & Ál tuquerque & NM \\
\hline Uni tersty of 'virginia & Charlottesuile & W厶 \\
\hline Weterans मे dministration Hospital - Ält & Al tuquerque & Now \\
\hline 'miake Forest IIni wersity-Eaptist Mledio & 'unston-Salem & No \\
\hline
\end{tabular}

